\title{
Decellularization of Human Internal Mammary Artery: Biomechanical Properties and Histopathological Evaluation
}

\author{
Abdol-Mohammad Kajbafzadeh, ${ }^{*}$ Reza Khorramirouz, Seyede Maryam Kameli, Javad Hashemi, and Amin Bagheri
}

\begin{abstract}
This study undertook to create small-diameter vascular grafts and assess their structure and mechanical properties to withstand arterial implantation. Twenty samples of intact human internal mammary arteries (IMAs) were collected and decellularized using detergent-based methods. To evaluate residual cellular and extracellular matrix (ECM) components, histological analysis was performed. Moreover, collagen typing and ECM structure were analyzed by Picrosirius red and Movat's pentachrome staining. Scanning electron microscopy was also applied to assess microarchitecture of both endothelial and adventitial surfaces of native and decellularized arterial samples. Furthermore, mechanical tests were performed to evaluate the rigidity and suture strength of the arteries. Human IMAs were completely decellularized in all three segments (proximal, middle, and distal). ECM proteins such as collagen and elastic fibers were efficiently preserved and no structural distortion in intima, media, and adventitial surfaces was observed. The parameters of the mechanical tests revealed no significant differences in the mechanical properties of decellularized arteries in comparison to native arteries with considerable strength, suture retention, and stress relaxation (Young's modulus $[\mathrm{MPa}$ ] $=0.22 \pm 0.023$ [native] and $0.22 \pm 0.015$ [acellular]; and suture strength $0.56 \pm 0.19$ [native] vs. $0.56 \pm 0.12$ [acellular], respectively). Decellularized IMA represents a potential arterial scaffold as an alternative to autologous grafts for future arterial bypass surgeries. By this technique, microarchitecture and mechanical integrity of decellularized arteries were considerably similar to native arteries. The goal of this study was to introduce an efficient method for complete decellularization of human IMA and evaluate the ECM and biomechanical properties.
\end{abstract}

Keywords: coronary artery disease; decellularization; internal mammary artery; revascularization; tissue engineering

\section{Introduction}

Cardiovascular diseases are one of the most common causes of death worldwide and the number of revascularization procedures is increasing considerably due to aging of the population. ${ }^{1}$ The internal mammary arteries (IMAs) are commonly used as the conduit of choice for myocardial revascularization owing to delayed development of atherosclerosis and greater long-term patency rate compared to saphenous vein grafts $\left(85 \%\right.$ vs. $61 \%$, respectively). ${ }^{2,3}$ A recent meta- analysis demonstrated that bilateral IMA grafting improves survival rate during the second postoperative decade in comparison with single IMA grafting. ${ }^{4} \mathrm{How}-$ ever, these grafts convey several limitations, including poor quality, limited length, vasospasm, and previous use. $^{5}$ Therefore, surgeons have turned to the use of the conventional vascular prostheses such as polyethylene terephthalate (Dacron) and expanded polytetrafluoroethylene (ePTFE) grafts. However, these prosthetic grafts are prone to thrombosis, inadequate mechanical

Section of Tissue Engineering and Stem Cell Therapy, Pediatric Urology and Regenerative Medicine Research Center, Children's Hospital Medical Center, Pediatric Center of Excellence, Tehran University of Medical Sciences, Tehran, Iran.

*Address correspondence to: Abdol-Mohammad Kajbafzadeh, MD, Section of Tissue Engineering and Stem Cell Therapy, Pediatric Urology and Regenerative Medicine Research Center, Children's Hospital Medical Center, Pediatric Center of Excellence, Tehran University of Medical Sciences, No. 62, Dr.Gharib's Street, Keshavarz Boulevard, Tehran, Iran 1419733151, E-mail: kajbafzd@sina.tums.ac.ir

(c) Abdol-Mohammad Kajbafzadeh et al. 2017; Published by Mary Ann Liebert, Inc. This is an Open Access article distributed under the terms of the Creative Commons Attribution License, which permits unrestricted use, distribution, and reproduction in any medium, provided the original work is properly cited. 
properties (compliance mismatch), intimal hyperplasia, and restenosis for small-diameter vessels $(<6 \mathrm{~mm})$, including coronary artery bypass grafts. ${ }^{6}$

To solve this problem, several biodegradable and biocompatible scaffolds seeding with vascular cells were developed. However, they have not practically achieved widespread acceptance because of some challenging issues, including inadequate initial strength, immunologic incompatibility, and delayed endothelialization, which may cause thrombosis and occlusion in low-flow conditions. ${ }^{7,8}$

Although vascular allografts showed high patency rates, their viable cells may result in acute immune rejection. Decellularization techniques by removing cells provide the proper microenvironment to support cell invasion, growth, and differentiation. Therefore, to provide vascular grafts with either immunoprivileged characteristics of autologous vessels or mechanical properties of native vessels, decellularized techniques should be developed as alternatives to autografts. ${ }^{9}$

In this study, we proposed that an optimized decellularization method of human IMA graft would preserve its structural integrity and mechanical properties to withstand arterial implantation.

\section{Materials and Methods}

\section{Sample preparation}

The Tehran University of Medical Sciences Ethics Committee approved this study. Twenty samples of human IMA were harvested bilaterally from braindeath patients (men younger than 50 years) during organ transplantation. These samples were collected from its origin in the subclavian artery to its bifurcation, which preserves proximal, middle, and distal segments of IMA (Fig. 1A). Harvested IMAs were gently dissected from surrounding adipose tissue and fascia, cannulated with a 14-gauge catheter at proximal site, and subsequently placed in heparinized normal saline solutions containing antibiotics (penicillin, gentamicin, ceftriaxone, and amphotericin B) at room temperature. Then, IMAs were attached to the pulsatile pump at a flow rate of $60 \mathrm{~mL} / \mathrm{min}$. The arteries were randomly divided into a native control group and three decellularization groups according to the following protocols: (1) $0.5 \%$ sodium dodecyl sulfate (SDS) for $24 \mathrm{~h}$; (2) $0.5 \%$ SDS for $48 \mathrm{~h}$; and (3) $1 \%$ Triton $\mathrm{x} 100$ for $24 \mathrm{~h}$, following by $1 \%$ SDS for $24 \mathrm{~h}$. They were subsequently flushed 10 times in phosphate-buffered saline (PBS) for periods of $15 \mathrm{~min}$ each.
Histological examination

To compare and evaluate the effectiveness of decellularization protocols in removing cell components, both native and decellularized specimens were cut into sections of $3 \mathrm{~mm} \times 4 \mathrm{~mm}$ and fixed in $10 \%$ neutral buffered formalin (Merck, Darmstadt, Germany) with $\mathrm{PH}=7.4$ for $48 \mathrm{~h}$ at room temperature. Then, the samples were washed in distilled water, dehydrated in graded ethanol, paraffin embedded, and sectioned into 5-8 $\mu \mathrm{m}$ thicknesses. For hematoxylin and eosin (H\&E) staining, the cellularity in three layers (intima, media, and adventitia) was evaluated and cell nuclei were counted in four different randomized regions, and the mean number was subsequently recorded. Moreover, muscle fibers and collagen tissue were evaluated by Masson's trichrome stain.

Picrosirius red and Russell-Movat's (pentachrome) staining were also applied for the purpose of collagen typing and ECM structure. For Picrosirius red staining, paraffin-embedded blocks were cut at $5 \mu \mathrm{m}$. The sections were deparaffinized and hydrated in graded alcohol series. The slides were stained in Picrosirius red solution for $1 \mathrm{~h}$ and $0.1 \%$ Sirius red (Direct Red 80; SigmaAldrich-365548) in saturated picric acid, followed by washing in two changes of $0.5 \%$ acetic acid without counterstaining. Under polarized microscopy, type I collagen fibers were seen as thick, yellow-orange fibers, and strongly birefringent; however, type III fibers were seen as thin, greenish fibers, and weakly birefringent.

Different constituents of connective tissue are highlighted in pentachrome staining. Differentiation of collagen, elastin, muscle, mucin, and fibrin is also demonstrated with this technique. For Movat's pentachrome staining, paraffin-embedded blocks were cut at $5 \mu \mathrm{m}$, deparaffinized, and hydrated subsequently in graded alcohol series. Briefly, mucins were stained with Alcian blue $8 \mathrm{GS}$ and converted to insoluble blue pigments with alkaline alcohol; elastic fibers and nuclei were stained black by Verheoff s hematoxylin; fibrinoid, fibrin, and muscle were stained red by Crocein scarlet-acid fuchsin solution (Sigma-Aldrich-C8822 and MERCK, 5231, respectively); and finally, collagen and reticular fibers were stained yellow by Saffron (Sigma-Aldrich-S8381).

Images were taken using a Nikon digital camera DXM 1200. Photoshop 10.0 software (Adobe Systems, Inc., Mountain View, CA) and Image Pro (Image Pro Inc., Boston, MA) were applied for analyzing the images at each time point. Histological evaluations were performed by two expert pathologists who were blind to the design. 

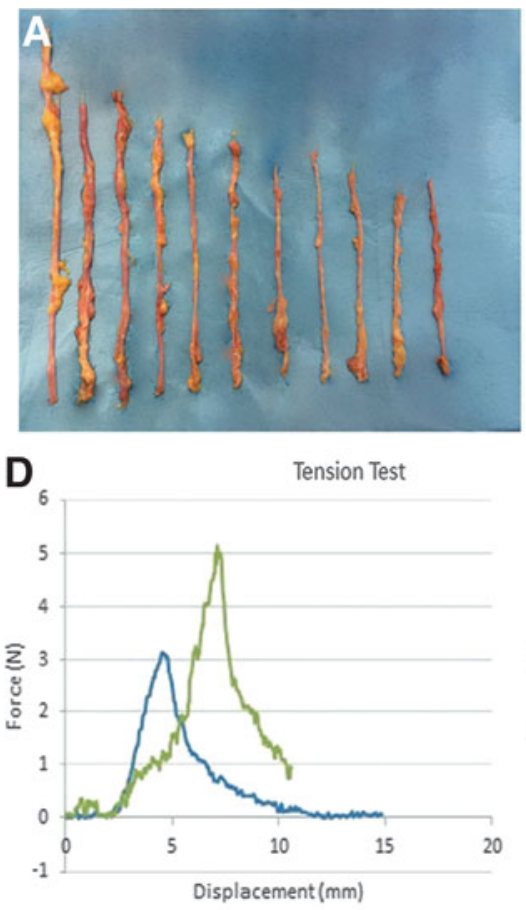

- Decellularized IMA — Naive IMA

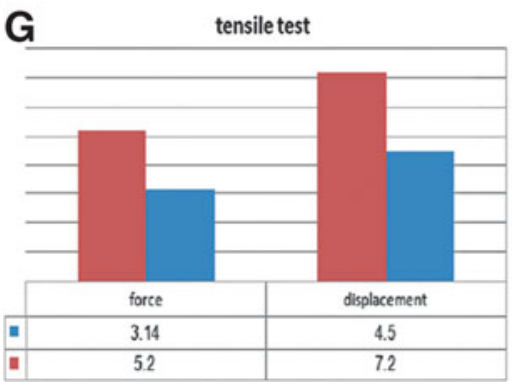

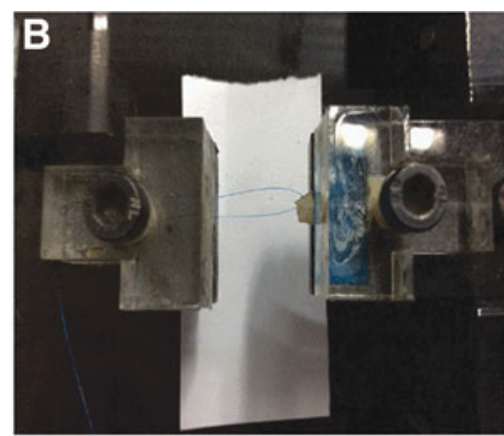
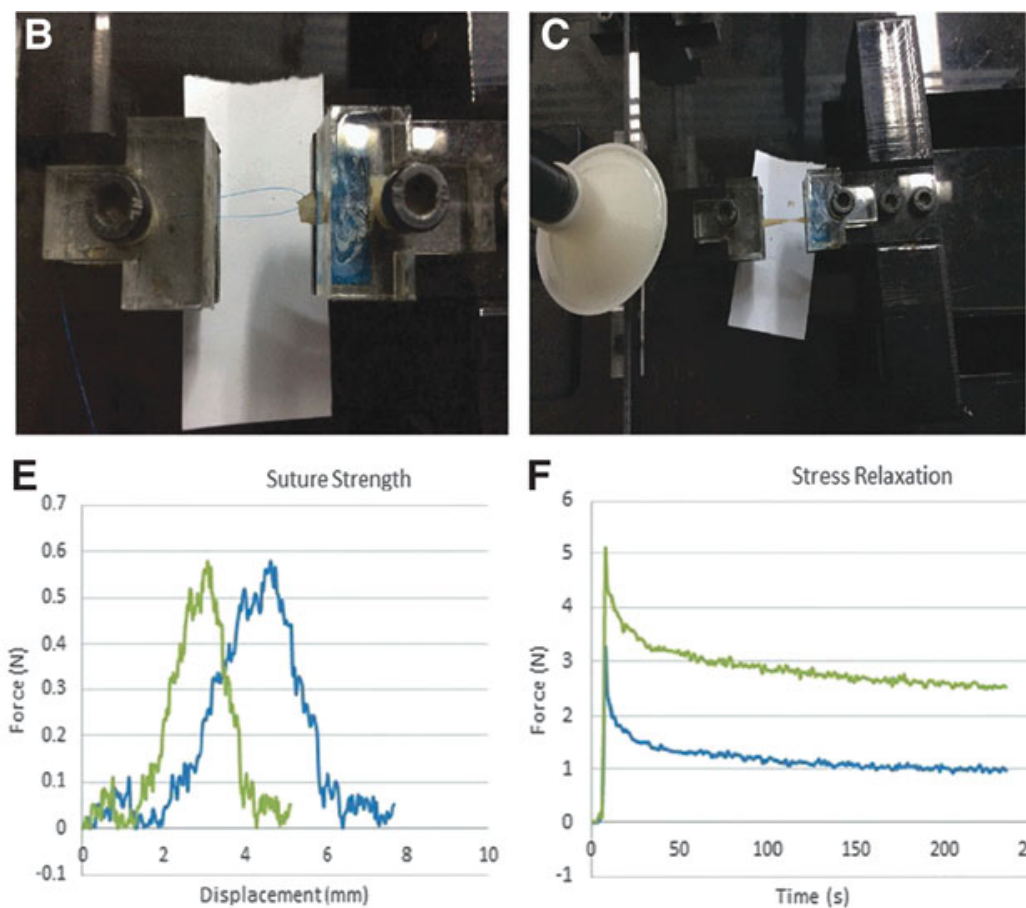

- Decellularized IMA - Naive IMA

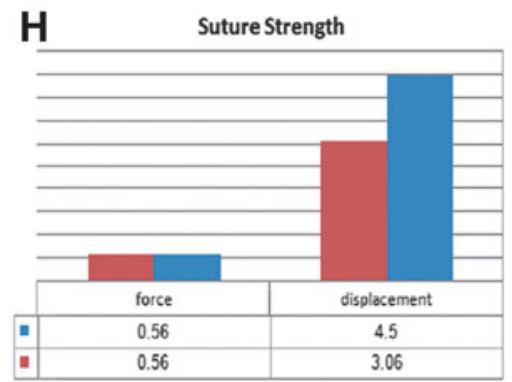

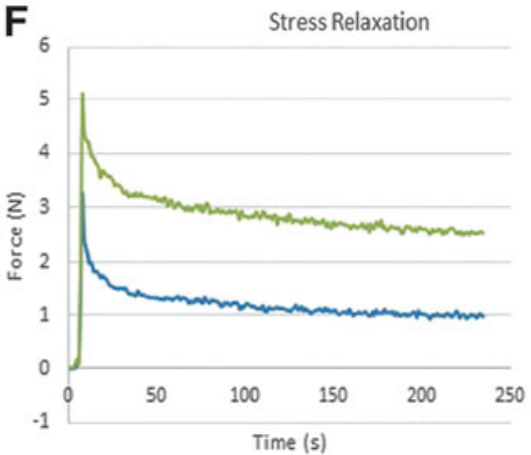

- Decellularized IMA Naive IMA

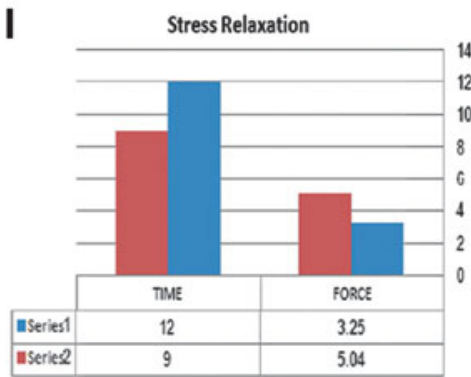

FIG. 1. (A) The IMAs. (B, C) Uniaxial test device for measuring mechanical properties such as suture strength and tension test. (D-I) Comparison of mechanical properties of decellularized and native IMAs. IMAs, human internal mammary arteries.

\section{DNA Quantification Test}

$50 \mathrm{mg}$ of acellular and native human IMA was immersed in the TE buffer to extract total DNA with the Genomic DNA purification Kit (Thermo Scientific, Lithuania). The concentration of DNA in the solution was quantified by spectrophotometry using NanoDropTechnology (Thermo scientific Nanodrop 1000). Then, the agarose gel was used to examine DNA assessment triplex.

\section{Scanning electron microscopy}

All samples (either native or decellularized IMAs) destined for scanning electron microscopy (SEM) were initially cut transversely and each piece was subsequently longitudinally split. Through this method, both endothelial and adventitial surfaces of the IMA were simultaneously exposed and evaluation was performed for both surfaces. Then, samples were fixed with $2.5 \%$ glutaraldehyde (Merck, Darmstadt, Germany) and washed thrice for 60 min each with PBS to wash out the remaining glutaraldehyde. Samples were completely dried using serial concentrations of $30 \%, 50 \%, 70 \%, 90 \%$, and $100 \%$ ethanol (Merck), each for $10 \mathrm{~min}$, and they were subsequently held under the hood overnight. Finally, gold sputter coating was applied to improve the conductivity of electrons 
and visualize the surfaces by SEM (Hitachi-54160, Japan). Then, all the samples were examined in a Jeol JSM-6340F Field Emission SEM. Acceleration voltage was adjusted at $10 \mathrm{kV}$ and a working distance of $8 \mathrm{~mm}$ was applied. Secondary and backscattered electrons were used for obtaining the images.

All scanning electron microscopic examinations as well as histological evaluations were performed for proximal, middle, and distal parts of IMAs (both native and decellularized samples), separately.

\section{Mechanical properties}

An in-house uniaxial test device, designed and constructed for measuring mechanical properties of soft biological tissues, was used to compare the tension tests, suture strength, and stress relaxation of native and decellularized IMAs (Fig. 1B, C). During the experiment, the specimens were sprayed with normal saline solution to preclude drying of the arteries. Sandpaper was used between the arteries and the jaw of the clamp to prevent slipping of the arteries. To evaluate the rigidity of the IMAs, tension test was performed with a constant elongation $(6 \mathrm{~mm} /$ min) along the longitudinal axis of the samples. Suture strength was measured using a 7.0 prolene su- ture placed in one side of the artery at $1.5 \mathrm{~mm}$ from the edge with a constant elongation as well as tension test. Furthermore, stress relaxation was done to evaluate the viscoelasticity property of the arteries. Stress relaxation is a decrement to stress in response to the constant amount of strain produced in the scaffold. For this purpose, the structure was held in a strained condition for a period of time.

\section{Statistical analysis}

All statistical analyses were performed by SPSS version 16.0 (SPSS Inc., Chicago, IL) software program. Each test was performed at least four times in room temperature and the average results were calculated. The data are expressed as mean \pm standard deviation (SD). Student's $t$-test was performed to evaluate comparative values. A $p$-value less than 0.05 was considered to have statistical significance.

\section{Results}

Treatment of IMA with protocol three (1\% Triton x100 for $24 \mathrm{~h}$ followed by $1 \%$ SDS for $24 \mathrm{~h}$ ) achieved complete decellularization of the arteries. However, the IMAs treated with protocols one and two were not as efficient as protocol three in removing cells.
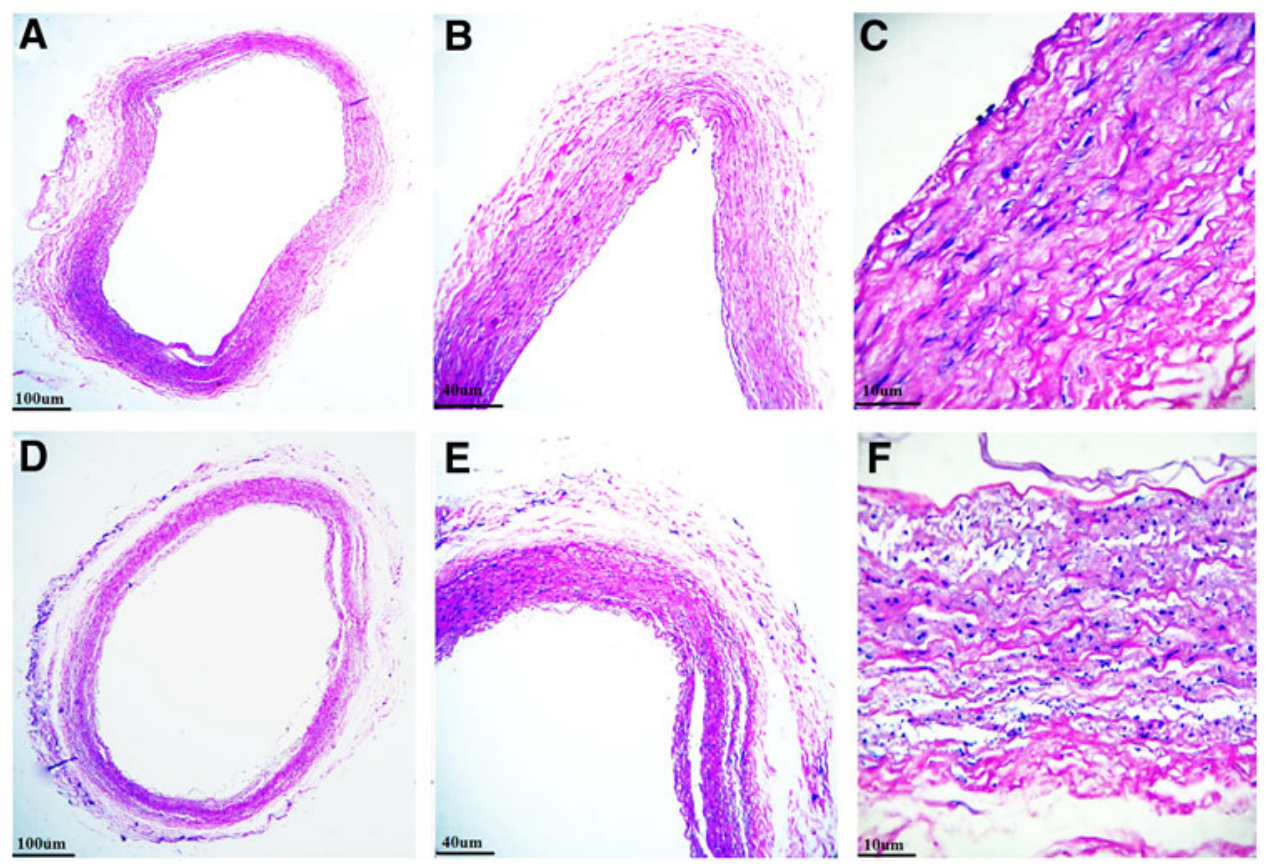

FIG. 2. H\&E staining of decellularized IMAs using protocol one (A-C) and 2 (D-F) showing presence of intact cells and cell remnants in samples treated with both protocols. H\&E, hematoxylin and eosin. 
Examination of native IMAs by H\&E staining demonstrated that intima and media comprised endothelial cells and smooth muscle cells (SMCs) with elastic tissue, respectively. Although intact cells and cell remnants were observed in samples treated with protocols one and two, complete decellularization accompanied by no endothelial lining and no nucleus in intima and medial layers was seen in all three segments of IMAs (proximal, middle, and distal) treated with protocol three (Figs. 2 and 3). Accordingly, protocol three was chosen for further evaluations. DNA quantification study result confirmed the successful decellularization of acellular sample compared to native sample (Fig. 4).

Masson's trichrome of the native IMAs showed that SMCs and collagen (stain red and blue, respectively) composed the main part of media and adventitia, respectively, in both proximal and distal segments. Examination of decellularized IMAs revealed intact vessel wall, lack of SMCs, and preserved collagen fibers accompanied by no structural distortion, in all three segments (Fig. 5).

The muscle, elastic, and collagen fibers were stained by Movat's pentachrome (stain red, black, and yellow, re- spectively). Proximal and distal segments of native IMAs are mainly composed of SMCs lining over media; however, Movat's pentachrome revealed more abundant elastic fibers in the middle segment. Moreover, distinct wavy internal and external elastic laminas present in all segments. After decellularization, all the SMCs were completely removed. Also, elastic and collagen fibers were preserved without structural distortion (Fig. 6).

Picrosirius red staining of native IMAs showed the greater concentration of collagen fibers in the adventitia and SMCs concomitant with elastin in the media. The collagen was preserved in all segments after decellularization (Fig. 7).

SEM examination of native IMAs demonstrated intact ultrastructures of either endothelial or adventitial surfaces of IMAs. Examination of decellularized IMAs revealed no cellularity or structural distortion in both surfaces of all three segments (Figs. 8 and 9).

The parameters of the tension test, suture strength, and stress relaxation revealed no significant changes in the mechanical properties between native and decellularized IMAs (Fig. 1D-F). Tension analysis for evaluating the

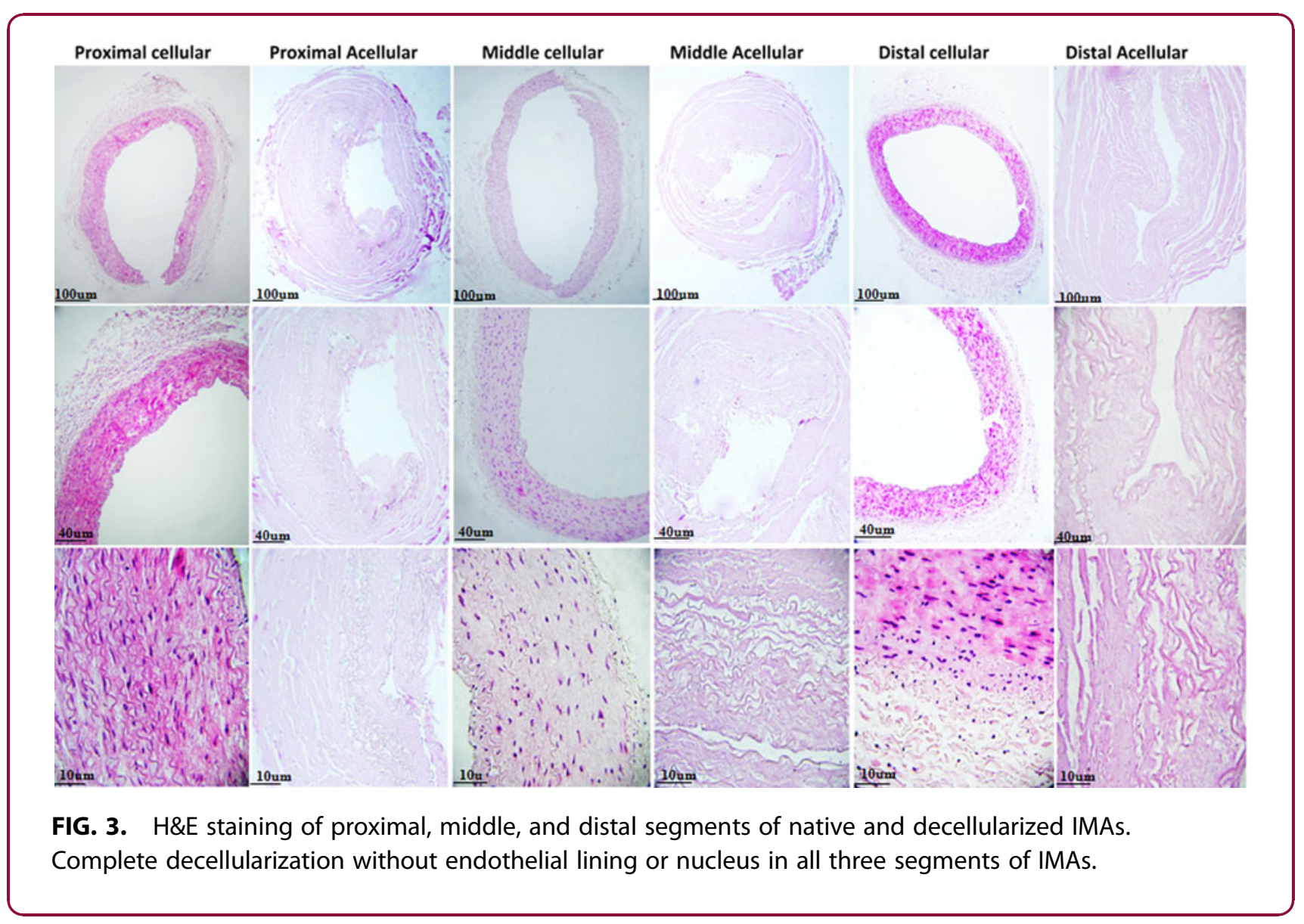




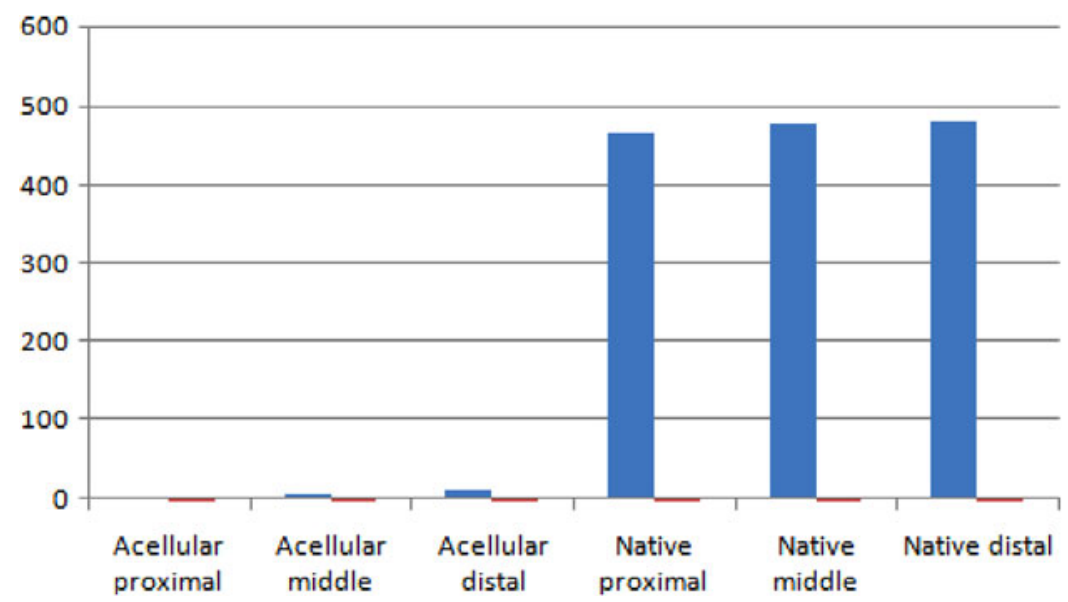

FIG. 4. DNA quantification for proximal, middle, and distal human IMAs (native and acellular).

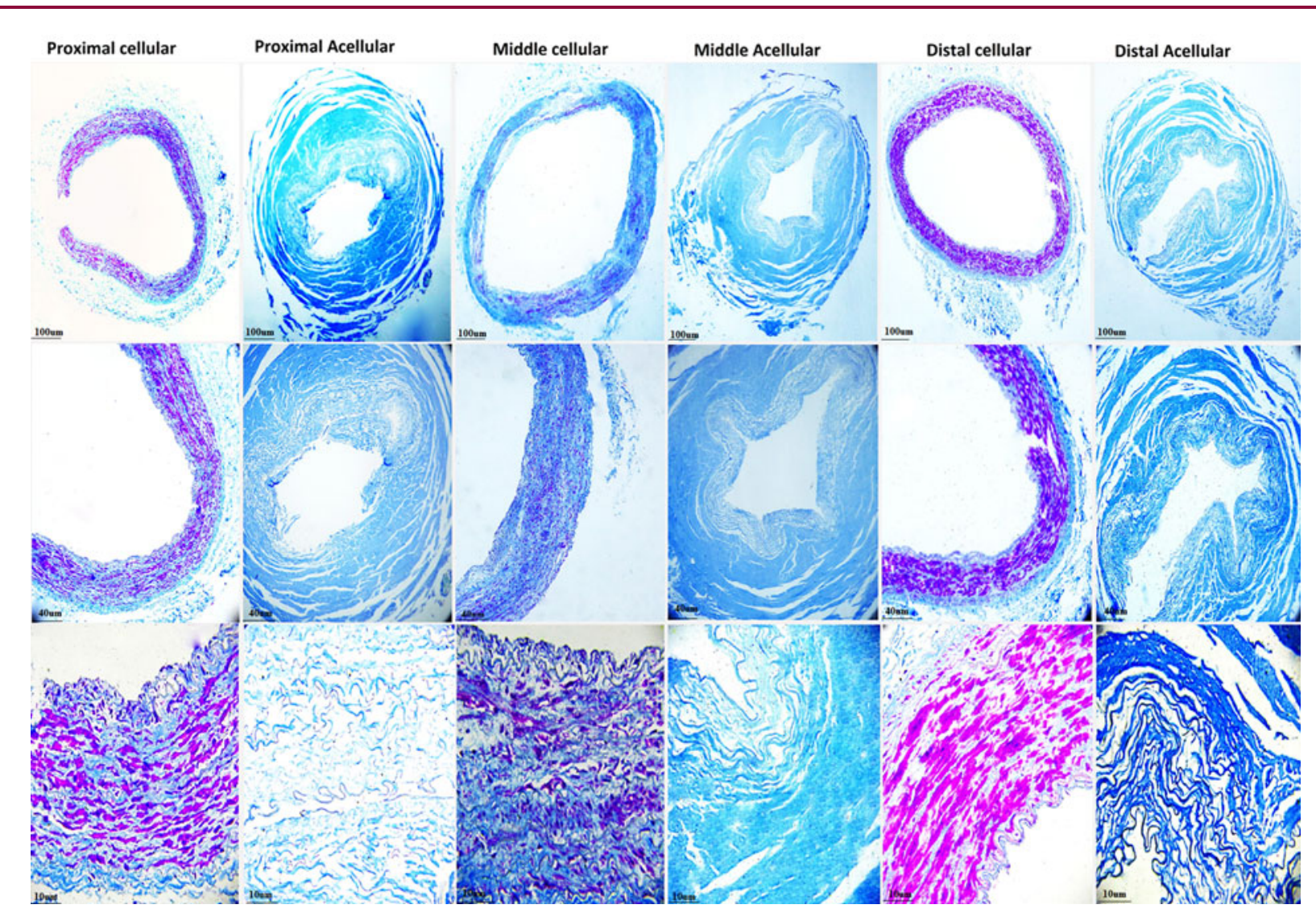

FIG. 5. Masson's trichrome staining of native and decellularized IMAs. Intact vessel wall, lack of smooth muscle cells, and preserved collagen fibers without structural distortion in all three segments of IMAs after decellularization. 


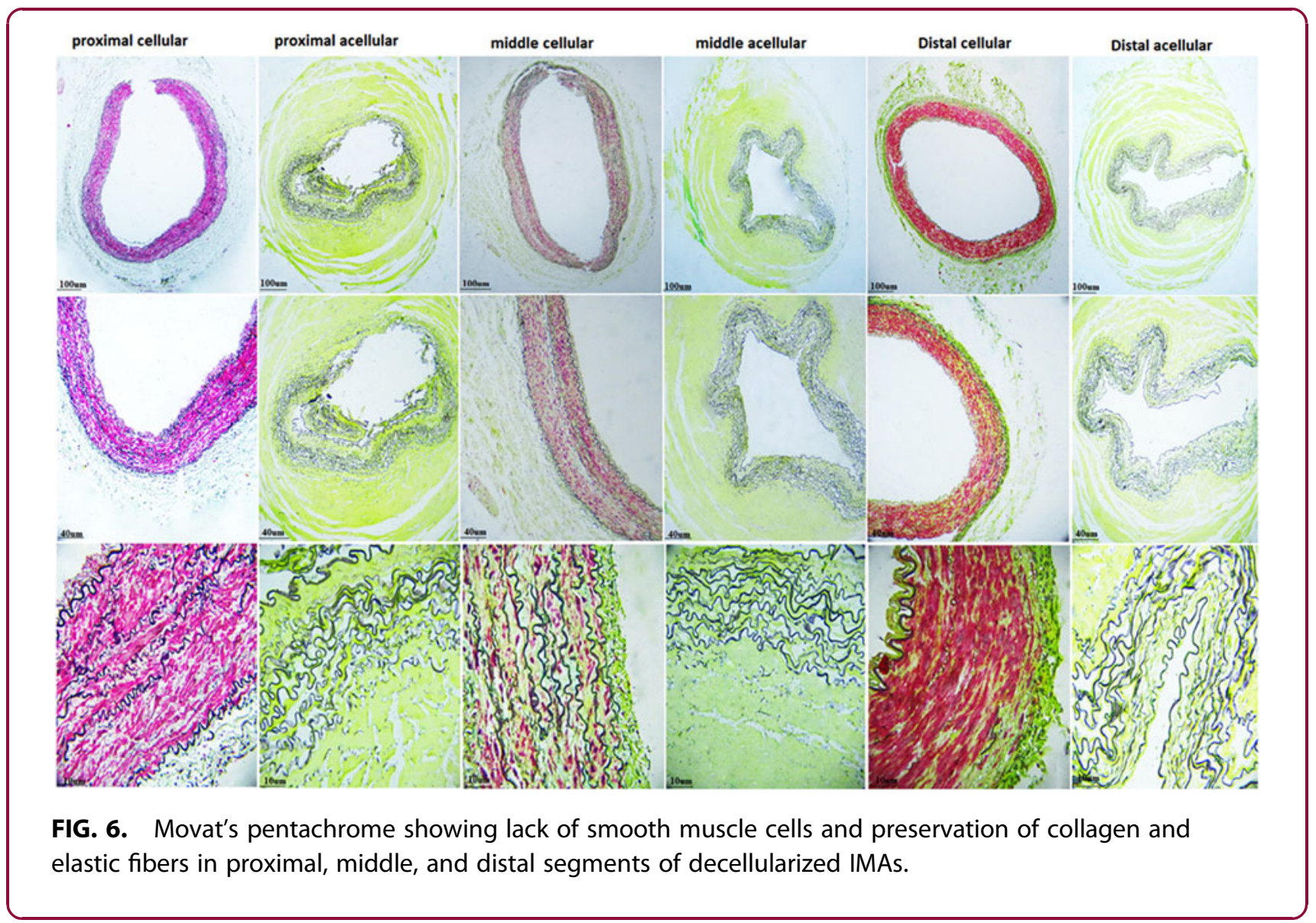

resistance to biomechanical forces demonstrated no significant differences between native and decellularized IMAs (Young's modulus $[\mathrm{MPa}]=0.22 \pm 0.023$ and $0.22 \pm 0.015$, respectively; $p>0.05$ ). Results of the suture strength revealed that there is no significant difference in the maximal force $(\mathrm{N})$ of the decellularized IMAs when compared to the native artery $(0.56 \pm 0.12$ vs. $0.56 \pm 0.19$, respectively). These data suggest that the decellularized IMA has sufficient suture retention strength.

\section{Discussion}

This study provided for the first time an intact decellularized IMA with efficient microarchitecture and mechanical integrity. Complete decellularization with preservation of ECM strength and morphology was consistently present using $1 \%$ Triton x100 followed by $1 \%$ SDS for $24 \mathrm{~h}$ each. Our results revealed that decellularized IMA can serve as an ideal scaffold for small-diameter vascular grafts.

About $50 \%$ of deaths from cardiovascular disease occur due to coronary artery occlusion. ${ }^{1}$ Over 700,000 coronary artery bypass graft surgeries are performed every year to restore blood flow distal to an arterial occlusion, mainly because of atherosclerosis. ${ }^{10}$ Although autologous arterial and venous grafts are the best options as bypass graft material, about $40 \%$ of patients requiring arterial bypass surgery do not have autologous grafts of sufficient length or quality. ${ }^{9}$ Moreover, only IMA grafts have shown promising results with high long-term patency rate, while using venous grafts, including the saphenous vein, can frequently lead to graft occlusion due to in vivo remodeling such as compliance mismatch and intimal hyperplasia. ${ }^{11,12}$ Therefore, alternative grafts are clearly needed.

Synthetic grafts, including Dacron and ePTFE, which work efficiently for large-diameter vascular grafts, have shown disappointing clinical results in small-diameter artery reconstruction with $20 \%$ decreased 5-year patency rate due to thrombotic complications when compared to venous bypasses. ${ }^{13}$ Despite their poor performance, none of these grafts is cost-effective. ${ }^{1}$ Tissue engineering has developed vascular grafts by taking advantage of biodegradable polymers such as polycaprolactone, polyglycolic acid, and polylactic acid. However, because of incompatibility with arterial remodeling, thrombus 


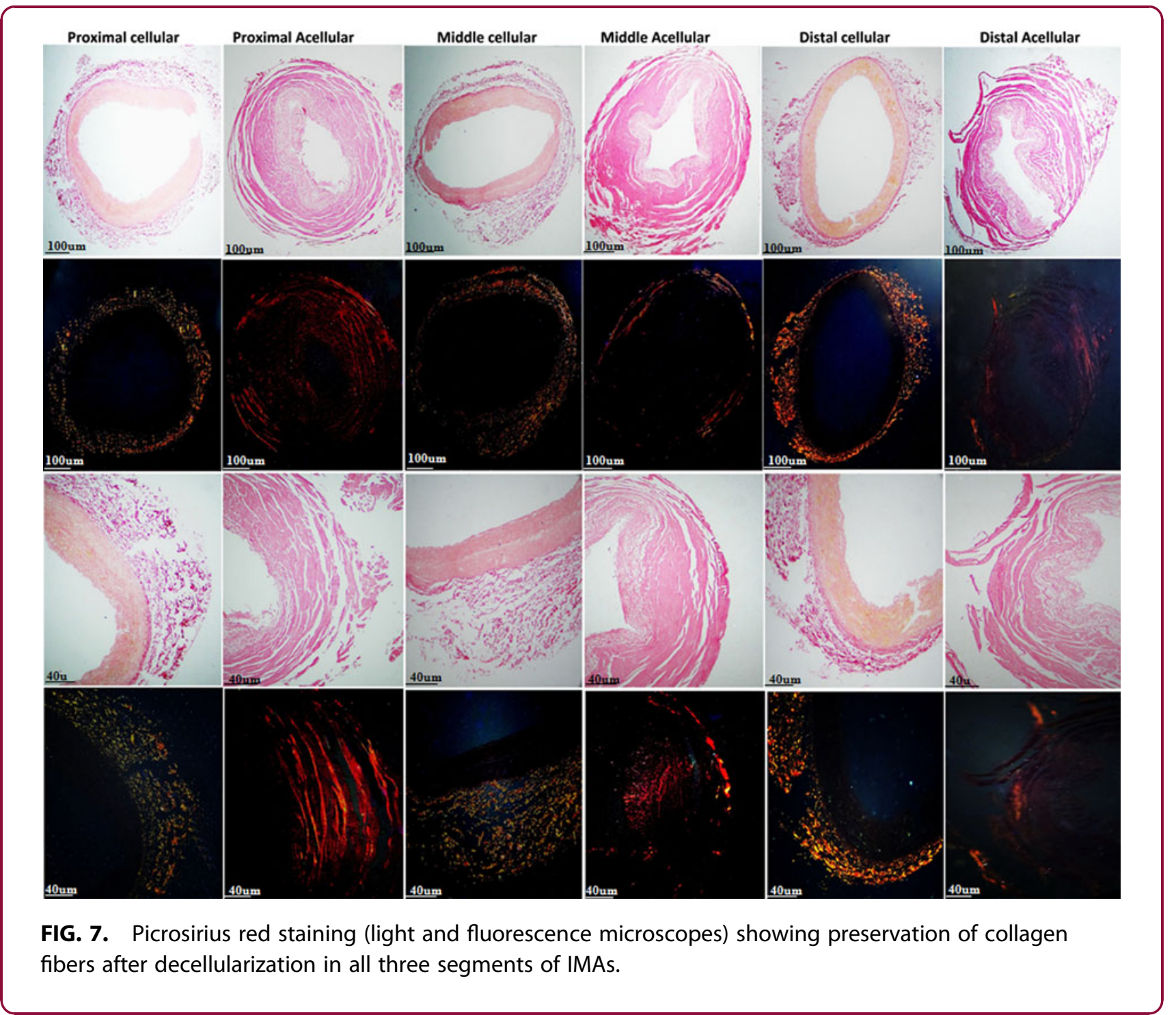

formation, and hyperplasia, these scaffolds have not produced ideal grafts for small-diameter vascular reconstructive surgery. ${ }^{5,8}$

Decellularization technique can play an important role in vascular tissue engineering by reducing antigenicity and subsequently graft rejection, while the architecture remains intact. ${ }^{1}$ Zou and Zhang decellularized efficiently porcine thoracic aorta with either Triton $\mathrm{x} 100$ or SDS solution. ${ }^{14}$ They showed that the structure of ECM was preserved. Sodium hydroxide and Triton x100 following by SDS were used to decellularize ovine coronary arteries during $74 \mathrm{~h} .{ }^{15}$ In a recent study, sodium hydroxide was able to remove cells from porcine coronary arteries in a process that took $9 \mathrm{~h} .{ }^{16}$ Although decellularized vessels have been previously reported, the use of this technique for human small-diameter vessels is still limited. Schaner et al. showed a decellularized human saphenous vein using $0.1 \%$ SDS for $15 \mathrm{~h}$ at $37^{\circ} \mathrm{C}^{17}$ They revealed that decellularized grafts have intact collagen morphology; however, their elastin staining decreased. Moreover, they found that the mechanical properties of decellularized vein such as suture strength were similar to native vein. Recently, Jones et al. placed the side branch of human left IMA in different concentrations of SDS for $2 \mathrm{~h}^{18}$ They revealed that complete decellularization was achieved using $0.15 \%$ and $0.3 \%$ SDS; however, loss of extracellular structure was seen at $0.3 \%$ SDS. Besides, its mechanical properties were not discussed in that study.

We developed a technique to decellularize all the segments of the human IMA, the artery that is documented for clinical use over decades, unlike its side branches, using Triton x100 and SDS, and 

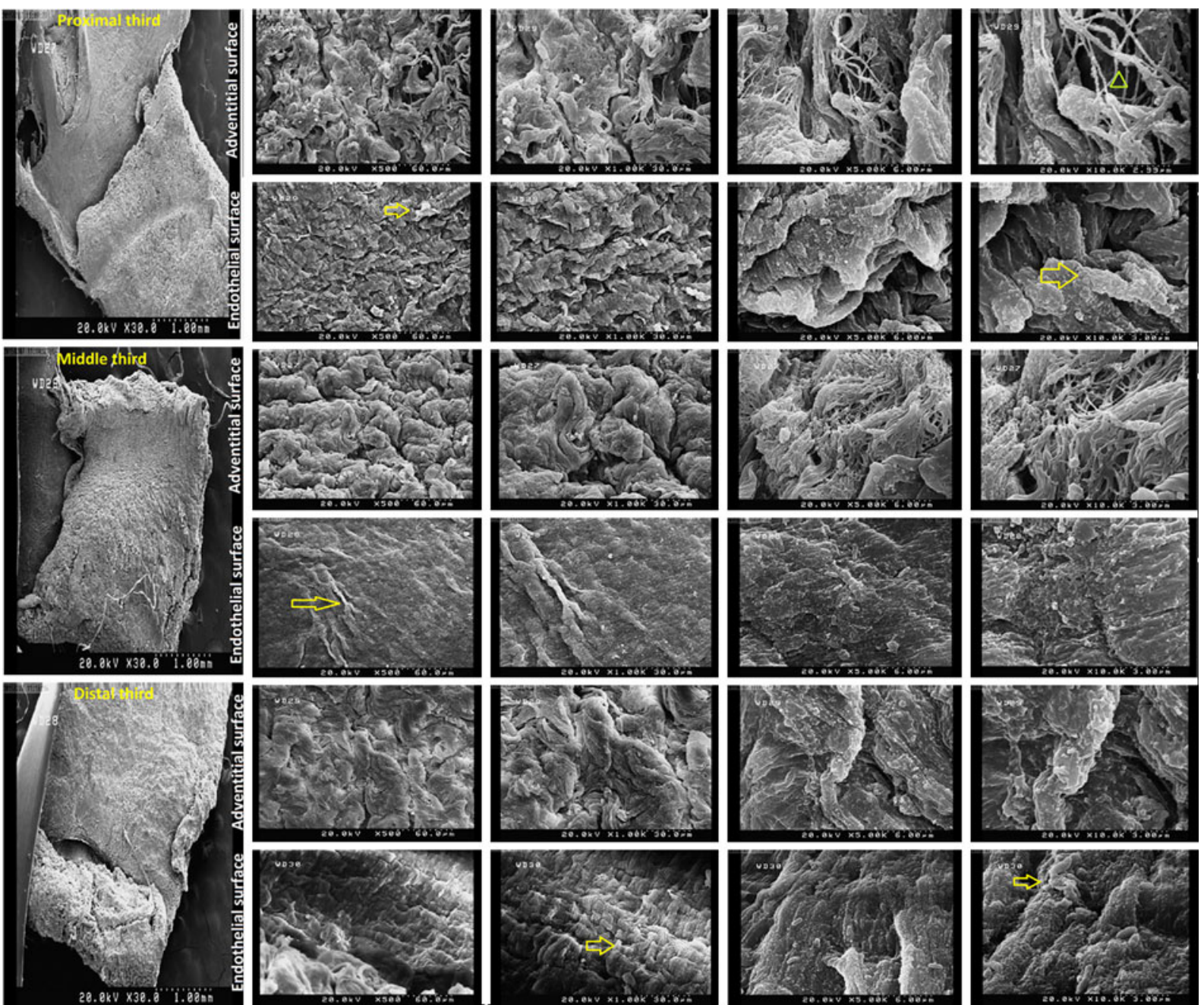

FIG. 8. Scanning electron microscopy showing 3-D microarchitecture of both endothelial and adventitial surfaces of native internal mammary arteries (yellow arrow: cell and green arrow head: fiber) (magnification of $500,1000,5000$, and 10000).

subsequently demonstrated preservation of ECM structure and mechanical integrity. Using human IMA offers several advantages because of its superiority over all other scaffolds, specifically the saphenous vein, in myocardial revascularization, owing to high longterm patency. ${ }^{2}$ The main goal of this study was to create a small-diameter vascular graft by decellularizing this valuable conduit and making it off-the-shelf for arterial bypass operations. We also assessed all segments of IMA (proximal, middle, and distal) to evaluate the efficacy of this method (Triton x100 and SDS solutions) to decellularize this graft completely and preserve its structure throughout these segments. In the study by Kneubil et al., the sequential histological structure of native IMA was examined. ${ }^{19}$ They found that the thickness of both intima and media layers was increased in the proximal segments, while elastin concentration was greater in the middle segments.

Decellularization with Triton x100 and SDS not only resulted in complete endothelial cell removal but also revealed lack of SMCs through all the segments of IMA. The efficacy of this technique may be because of the fact that Triton $\mathrm{x} 100$, a nonionic surfactant, as the first solution is efficient to dissolve cell membranes by disrupting lipidprotein and lipid-lipid interactions. ${ }^{20}$ While SDS as an anionic detergent is used to clear the residue nuclear remnants from the matrix after using Triton $\times 100,{ }^{21,22}$ our results revealed the effect of Triton $\mathrm{x} 100$ when it was used 

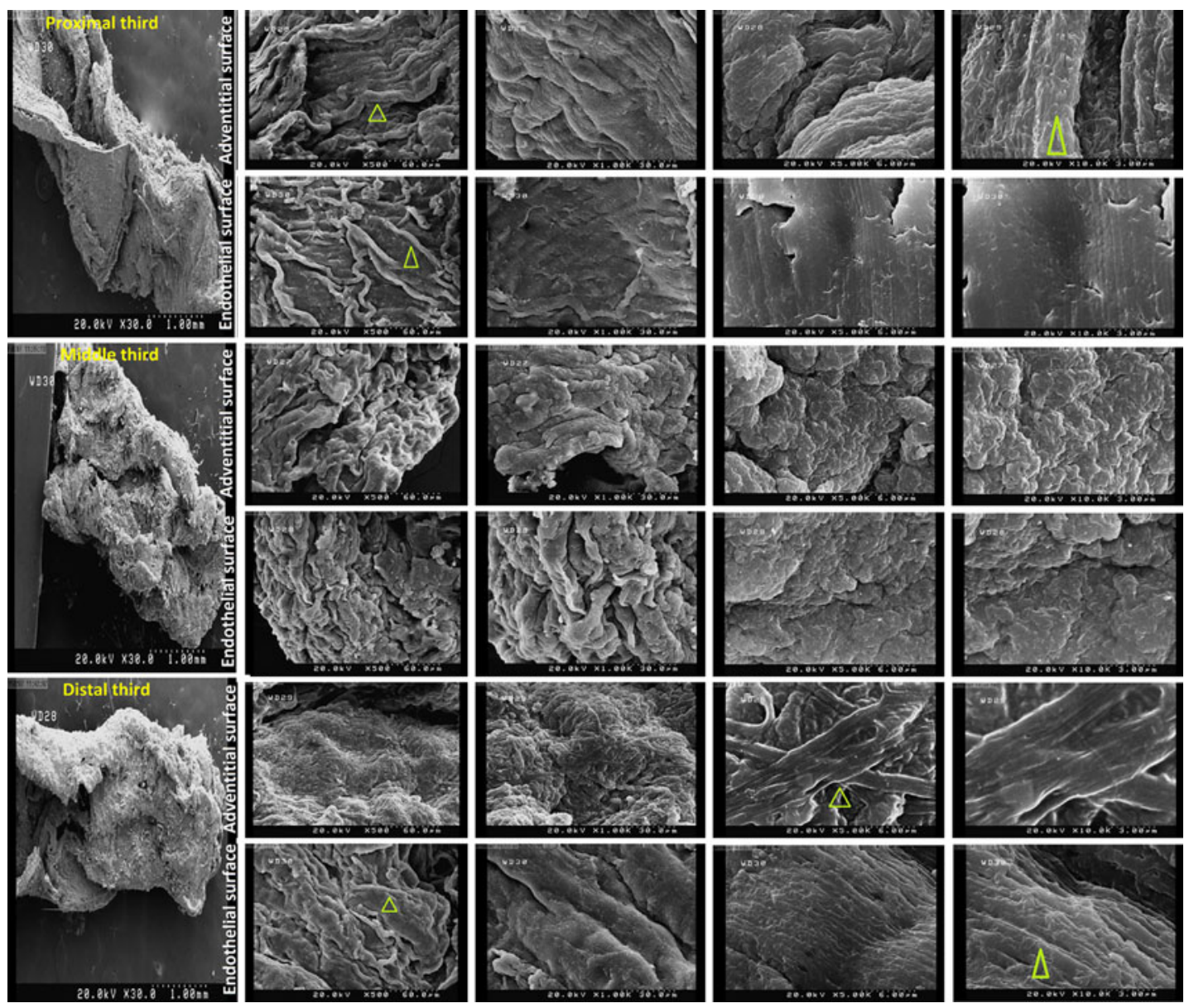

FIG. 9. Scanning electron microscopy of proximal, middle, and distal segments of decellularized IMAs. Intact ultrastructure accompanied by no cellularity in either endothelial or adventitial surfaces of all three segments (yellow arrow: cell and green arrow head: fiber) (magnification of 500, 1000, 5000 and 10000).

before SDS (protocol three) compared to SDS alone (protocols one and two). Histological analysis of decellularized IMA showed intact natural microstructure and preserved ECM. This result is consequential because elastic fibers are responsible for distensibility and recoil, and collagen supplies vessel strength. ${ }^{23}$

The SEM plays a significant role in evaluating the conservation of microarchitecture and ECM proteins such as elastin and collagen. The integrity of preservation of ECM proteins and intact structure of decellularized IMA was considerably similar to native artery. This finding is important as efficient conservation of elastin and collagen may provide better cell signaling and differentiation after in vivo implantation. ${ }^{22}$

Any arterial bypass graft needs adequate initial strength. Gui et al. showed no significant change in the mechanical strength of the decellularized umbilical artery in comparison to its native form. ${ }^{24}$ In another study, Zou and Zhang revealed that decellularized porcine thoracic aorta using SDS and Triton x-100 exhibits similar elastic properties as native aorta; however, a decellularized graft shows less stress relaxation than a native one. ${ }^{14}$ One of the goals of this study was to assess the mechanical properties such as tension test, suture strength, and stress relaxation of decellularized IMA. Our results suggest that mechanical properties of IMAs treated with Triton and SDS solutions did not significantly differ from native grafts. Consequently, treated IMAs were utilized in arterial circulation with considerable strength, suture retention, and stress relaxation.

Although this study shows promising results to use decellularized IMAs as small-diameter vascular grafts, 
lack of an in vivo study is one of its limitations. This initial study tries to evaluate the patency of decellularized human IMAs as an alternative to autologous grafts in arterial revascularization; however, animal studies must be applied to assess the efficiency of this arterial conduit before clinical practice. Another limitation for this study is lack of evaluation of MHC 1 quantification to ascertain the removal of immunogenic properties of these particles.

\section{Conclusions}

We have shown that decellularized human IMA using Triton $\mathrm{x}-100$ followed by SDS represents a potential arterial scaffold as an alternative to autologous grafts for future arterial bypass surgeries. This demonstrates that microarchitecture and mechanical integrity of decellularized IMA were considerably similar to native artery.

\section{Acknowledgment}

The grant was funded by Tehran University of Medical Science.

\section{Author Disclosure Statement}

No competing financial interests exist.

\section{References}

1. Seifu DG, Purnama A, Mequanint K, et al. Small-diameter vascular tissue engineering. Nat Rev Cardiol. 2013;10:410-421.

2. Goldman S, Zadina K, Moritz T, et al. Long-term patency of saphenous vein and left internal mammary artery grafts after coronary artery bypass surgery: results from a Department of Veterans Affairs Cooperative Study. J Am Coll Cardiol. 2004;44:2149-2156.

3. Otsuka F, Yahagi K, Sakakura K, et al. Why is the mammary artery so special and what protects it from atherosclerosis? Ann Thorac Surg. 2013;2:519-526.

4. Yi G, Shine B, Rehman SM, et al. Effect of bilateral internal mammary artery on long-term survival: a meta-analysis approach. Circulation. 2014;130:539-545.

5. Pektok E, Nottelet B, Tille JC, et al. Degradation and healing characteristics of small-diameter poly ( $\varepsilon$-caprolactone) vascular grafts in the rat systemic arterial circulation. Circulation. 2008;118:2563-2570.

6. Pawlowski KJ, Rittgers SE, Schmidt SP, et al. Endothelial cell seeding of polymeric vascular grafts. Front Biosci. 2004;9:1412-1421

7. Innocente F, Mandracchia D, Pektok E, et al. Paclitaxel-Eluting biodegradable synthetic vascular prostheses a step toward reduction of neointima formation? Circulation. 2009:120(suppl 1):S37-S45.

8. Patterson JT, Gilliland T, Maxfield MW, et al. Tissue-engineered vascular grafts for use in the treatment of congenital heart disease: from the bench to the clinic and back again. Regen Med. 2012;7:409-419.

9. Moroni F, Mirabella T. Decellularized matrices for cardiovascular tissue engineering. Am J Stem Cells. 2014;3:1.

10. Ramesh B, Mathapati S, Galla S, et al. Crosslinked acellular saphenous vein for small-diameter vascular graft. Asian Cardiovasc Thorac Ann. 2013;21:293-302.

11. Liu S, Moore M, Yap C. Prevention of mechanical stretch-induced endothelial and smooth muscle cell injury in experimental vein grafts. J Biomech Eng. 2000;122:31-38.

12. Meng X, Mavromatis K, Galis ZS. Mechanical stretching of human saphenous vein grafts induces expression and activation of matrixdegrading enzymes associated with vascular tissue injury and repair. Exp. Mol. Pathol. 1999;66:227-237.

13. Klinkert $P$, Post $P$, Breslau $P$, et al. Saphenous vein versus PTFE for aboveknee femoropopliteal bypass. A review of the literature. Eur J Vasc Endovasc Surg. 2004;27:357-362.
14. Zou Y, Zhang Y. Mechanical evaluation of decellularized porcine thoracic aorta. J Surg Res. 2012;175:359-368.

15. Lopez-Soler Rl, Brennan MP, Goyal A, et al. Development of a mouse model for evaluation of small diameter vascular grafts. J Surg Res. 2007;139:1-6.

16. Campbell $E$, Cahill $P$, Lally C. Investigation of a small-diameter decellularised artery as a potential scaffold for vascular tissue engineering; biomechanical evaluation and preliminary cell seeding. J Mech Behav Biomed Mater. 2012;14:130-142.

17. Schaner PJ, Martin ND, Tulenko TN, et al. Decellularized vein as a potential scaffold for vascular tissue engineering. J Vasc Surg. 2004;40:146-153.

18. Jones SG, Hu Y, Xu Q, et al. Stem cells accumulate on a decellularized arterial xenograft in vivo. Ann Thorac Surg. 2014;97:2104-2110.

19. Kneubil MC, Gomes WJ, Aquino MS, et al. Sequential histomorphometric study of the left internal thoracic artery. Rev Bras Cir Cardiovasc. 2006;21:371-376.

20. De Kock J, Ceelen L, De Spiegelaere W, et al. Simple and quick method for whole-liver decellularization: a novel in vitro three-dimensional bioengineering tool? Arch Toxicol. 2011;85:607-612.

21. Ott HC, Matthiesen TS, Goh SK, et al. Perfusion-decellularized matrix: using nature's platform to engineer a bioartificial heart. Nat Med. 2008;14:213-221.

22. Sabetkish S, Kajbafzadeh AM, Sabetkish N, et al. Whole-organ tissue engineering: decellularization and recellularization of three-dimensional matrix liver scaffolds. J Biomed Mater Res A. 2015;103:1498-1508.

23. Douglas J, Gaughran E, Henderson J, et al. The use of segmental arterial implants prepared by enzymatic modification of heterologous blood vessels. Surg forum. 1956:6:242-246.

24. Gui L, Muto A, Chan SA, et al. Development of decellularized human umbilical arteries as small-diameter vascular grafts. Tissue Eng Part A. 2009;15:2665-2676

Cite this article as: Kajbafzadeh AM, Khorramirouz R, Kameli SM Hashemi J, Bagheri A (2017) Decellularization of human internal mammary artery: biomechanical properties and histopathological evaluation, BioResearch Open Access, 6:1, 74-84, DOl: 10.1089/ biores.2016.0040

$\begin{aligned} & \text { Abbreviations Used } \\ & \mathrm{IMA}=\text { internal mammary arteries } \\ & \mathrm{SEM}=\text { Scanning electron microscopy } \\ & \mathrm{ECM}=\text { extracellular matrix } \\ & \mathrm{ePTFE}=\text { expanded polytetrafluoroethylene } \\ & \mathrm{H \& E}=\text { hematoxylin and eosin } \\ & \mathrm{SMC}=\text { smooth muscle cells } \\ & \mathrm{MHC} 1=\text { major histocompatibility complex } 1\end{aligned}$

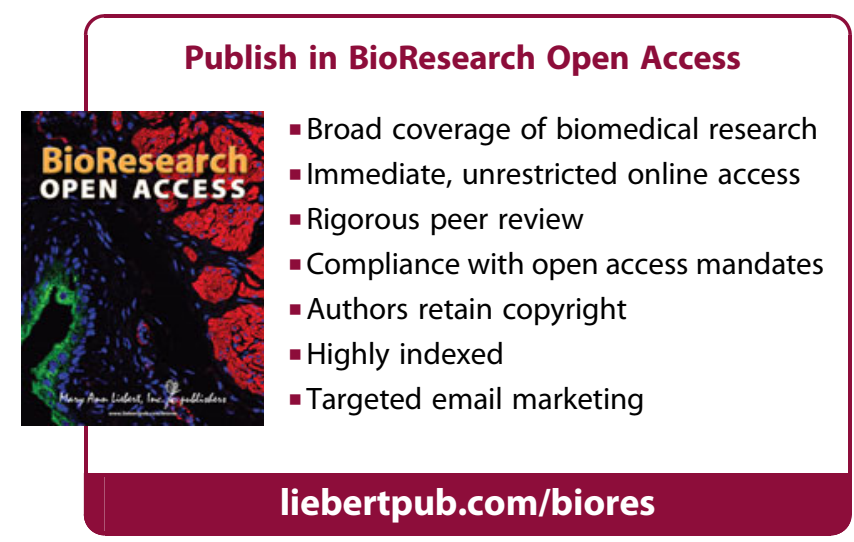

